\title{
Lipid Peroxidation Activity and Immune Response in Modeling Inflammatory and Degenerative Damage to the Periodontium of Rats
}

\author{
Marina A. Darenskaya, $\mathrm{PhD}, \mathrm{ScD}^{1 *}$; Lyudmila A. Grebenkina, $\mathrm{PhD}, \mathrm{ScD}^{1}$; \\ Evgeniy V. Mokrenko, $\mathrm{PhD}^{2}$; Petr D. Shabanov, $\mathrm{PhD}, \mathrm{ScD}^{3}$; Maria I. Suslikova, $\mathrm{PhD}^{2}$; \\ Mark E. Mokrenko ${ }^{2}$; Yulia O. Sinyova ${ }^{4}$; Ivan S. Goncharov²; Marina I. Gubina, $\mathrm{PhD}^{2}$; \\ Igor Yu. Kostritsky²; Alexandra F. Bulnaeva, $\mathrm{PhD}^{2}$; Elena V. Proskurnina, $\mathrm{PhD}, \mathrm{ScD}^{5}$; \\ Lyubov I. Kolesnikova, Academician of the RAS ${ }^{1}$; Sergey I. Kolesnikov, Academician of the RAS ${ }^{1,5}$ \\ ${ }^{1}$ Scientific Centre for Family Health and Human Reproduction Problems, Irkutsk, the Russian Federation \\ 'Irkutsk State Medical University, Irkutsk, the Russian Federation \\ ${ }^{3}$ Russian military medical Academy, St. Petersburg, the Russian Federation \\ ${ }^{4}$ Irkutsk National Research Technical University, Irkutsk, the Russian Federation \\ ${ }^{5}$ M.V. Lomonosov Moscow State University, Moscow, the Russian Federation
}

\begin{abstract}
The aim of this study was to assess changes in the lipid peroxidation activity and immune response in modeling inflammatory and degenerative damage to the soft tissues of the periodontium of Wistar rats.

Materials and Methods: The experiments were carried out on male Wistar rats weighing 220-250g. Modeling the inflammatory and degenerative damage to the soft tissues of the periodontium in animals $(n=10)$ of the test group (TG) was carried out using a single dose of a $2 \%$ formaldehyde aqueous solution injected into each side of the outer part of the gums $(0.15$ $\mathrm{ml})$ at the level of the lower molars of anesthetized animals. The control group (CG) of animals $(\mathrm{n}=10)$ received an injection of physiological saline solution in the same volume. In 3 days after damage modeling, the animals were killed by decapitation and blood samples were drawn for testing. The intensity of LPO processes in the blood was estimated by content of conjugated dienes and TBA-reactive substances (TBARS). The status of the AOD system was determined by the SOD activity and GSH level. The immune status of the animals was assessed by several indicators as leukocyte migration inhibition test (LMIT), the phagocytic reaction of neutrophils, and oxygen-dependent and oxygen-independent microbicidal activity of neutrophils.

Results: The results obtained showed that the modeling of inflammatory and degenerative damage to the periodontium in rats was characterized by significant changes, affecting both the LPO system and the immune response. An LMIT conducted with various mitogens demonstrated the development of immunodeficiency in TG characterized by high activity of the inflammatory process. Immune disorders in animals of TG were also supported by changes in the phagocytosis system. Low levels on the integral NBT test indicate disorders in the state of oxygen-dependent microbicidal systems of phagocytes. The assessment of the activity of oxygen-independent microbicidal systems of phagocytes in LCT also found disorders in the immune status of animals of TG.

Conclusion: Inflammatory and degenerative damage of the soft tissues of the periodontium in rats is accompanied by pronounced disorders in both the LPO system and the immune response.(International Journal of Biomedicine. 2019;9(2):159-162.)
\end{abstract}

Key Words: periodontitis $\bullet$ inflammation $\bullet$ rats $\bullet$ lipid peroxidation $\bullet$ phagocytes

\section{Abbreviations}

AOD, antioxidative defense; CD, conjugated dienes; ConA, concanavalin A; GSH, reduced glutathione; LMIT, leukocyte migration inhibition test; LPO, lipid peroxidation; LCT, lysosomal-cation test; NBT test, nitroblue tetrazolium reduction test; OS, oxidative stress; PI, phagocytic index; PN, phagocyte number; PHA, phytohemagglutinin; ROS, reactive oxygen species; SOD, superoxide dismutase; TBARS, TBA-reactive substances. 


\section{Introduction}

The Global Burden of Disease Study (1990-2010) indicates that severe periodontitis is the sixth most prevalent disease worldwide.(1) Periodontal diseases are responsible for 3.5 million years lived with disability. ${ }^{(2)}$ The overall prevalence of periodontitis increases with age, and the incidence rises steeply in adults aged $30-40$ years. ${ }^{(3)}$

As shown in a number of experimental and clinical works, under the influence of various types of factors a number of pathological changes with a predominance of inflammatory and destructive manifestations appear in the periodontium tissues. ${ }^{(4-6)}$ Periodontitis appears to have multiple etiologies with a microbial factor contributing to initiation of the disease and an immunological factor of the host propagating the disease. ${ }^{(7)}$ Processes of free-radical oxidation connected with phagocytes, producing ROS, fall into unspecific defense mechanisms. ${ }^{(8-13)}$ This evolved secretory function of phagocytes is necessary to eliminate bacteria. The intensive ROS generation that triggers LPO processes in cell biomembranes takes place under the conditions of oxygen overconsumption in phagocytosis. ${ }^{(14-16)}$ As a result of damage, severe disturbances arise in the regulation of the biomembrane state. Thus, abnormal proteins and secondary destructive processes appear, which cause profound disorders in cell membrane architecture and cell death. ${ }^{(17,18)}$ Despite numerous studies of redox-changes in the tissues of the periodontium, an interpretation of findings is still questionable. ${ }^{(17,19)}$ Thus, modeling inflammatory and degenerative damage in the soft tissues of the periodontium seems to be very important for clarifying the pathogenesis of periodontitis.

The aim of this study was to assess changes in the lipid peroxidation activity and immune response in modeling inflammatory and degenerative damage to the soft tissues of the periodontium of Wistar rats.

\section{Materials and Methods}

The experiments were carried out on male Wistar rats weighing 220-250g. All animals were given access to food and water ad libitum.

Modeling the inflammatory and degenerative damage to the soft tissues of the periodontium in animals $(n=10)$ of the test group (TG) was carried out using a single dose of a $2 \%$ formaldehyde aqueous solution injected into each side of the outer part of the gums $(0.15 \mathrm{ml})$ at the level of the lower molars of anesthetized animals. ${ }^{(5)}$ The control group (CG) of animals $(\mathrm{n}=10)$ received an injection of physiological saline solution in the same volume. In 24 hours, we found stable extensive inflammatory and degenerative changes in the soft tissues of the periodontium at the injection site. In 3 days after damage modeling, the animals were killed by decapitation and blood samples were drawn for testing.

The intensity of LPO processes in the blood was estimated by content of primary oxidation products (CD) and secondary oxidation products (TBARS) ${ }^{(20)}$ The status of the AOD system was determined by the SOD activity and GSH level. ${ }^{(20)}$
The immune status of the animals was assessed by several indicators as LMIT, the phagocytic reaction of neutrophils, and oxygen-dependent and oxygen-independent microbicidal activity of neutrophils. LMIT assessment methodology is based on the ability of lymphocytes to secrete leukocyte migration inhibitory factor in response to the nonspecific mitogens PHA and ConA (LMIT was expressed in \%). ${ }^{(21)}$ The phagocytic activity of neutrophils was assessed by phagocytic index (PI), which demonstrates phagocyte count from among calculated neutrophils, as well as phagocyte number $(\mathrm{PN})$ : the average number of microbes absorbed by one active neutrophil (RU). To analyze the digesting function, IPC (the ratio of the number of digested microbes to the average number of absorbed microbes, both digested and undigested) was defined (in \%).

The activity of oxygen-independent microbicidal systems of phagocytes was estimated by LCT (in \%). . $^{(21,22)}$ The principle of the test is based on cytochemical detection of nonenzyme lysosomal-cation proteins, whose relative count in studied cells indicates the presence of concerned antimicrobial systems. The activity of oxygen-dependent microbicidal systems of phagocytes was estimated by the NBT test (in \%). ${ }^{(21)}$ The NBT test checks if certain immune system cells can change a colorless chemical called nitroblue tetrazolium (NBT) into a deep blue color. In the NBT test, neutrophils change the colorless compound NBT into a compound with a deep blue color. If this test is negative (i.e., no blue color is produced), then this indicates a defect in superoxide-generating NADPH oxidase activity with inability to efficiently kill phagocytized bacteria. ${ }^{(22,23)}$ The NBT test is a qualitative assay of ROS production.

Experiment was performed in accordance with the Guide for the Care and Use of Laboratory Animals (The institute of Laboratory Animal Resources,1996); Guidance on experimental (non-clinical) testing of new pharmaceutical substances, 2005); ${ }^{(24)}$ Order of the Ministry of Health of the Russian Federation № 708n (23.08.2010) “On approval of the rules of Good Laboratory Practice» (GLP).

Statistical analysis was performed using the Statistica 6.1 software package (Stat-Soft Inc., USA). The normality of distribution of continuous variables was tested by the Kolmogorov-Smirnov test with the Lilliefors correction and Shapiro-Wilk test. For descriptive analysis, results are presented as median (Me), interquartile range (IQR; 25th to 75th percentiles). F-test for testing equality of variance was used to test the hypothesis of the equality of two population variances. For data with normal distribution, inter-group comparisons were performed using Student's t-test. Differences of continuous variables departing from the normal distribution, even after transformation, were tested by the Mann-Whitney U-test. A probability value of $P<0.05$ was considered statistically significant.

\section{Results and Discussion}

The assessment of the LPO reactions in rats with inflammatory and degenerative damage to the soft tissues of the periodontium (TG) found a statistically significant increase in the content of CD (by 3.56 times) and TBARS (by 5.82 
times) compared to intact animals (Fig.1). It was also noted that activity of SOD and GSH was reduced by 3.93 times and 3.41 times, respectively.

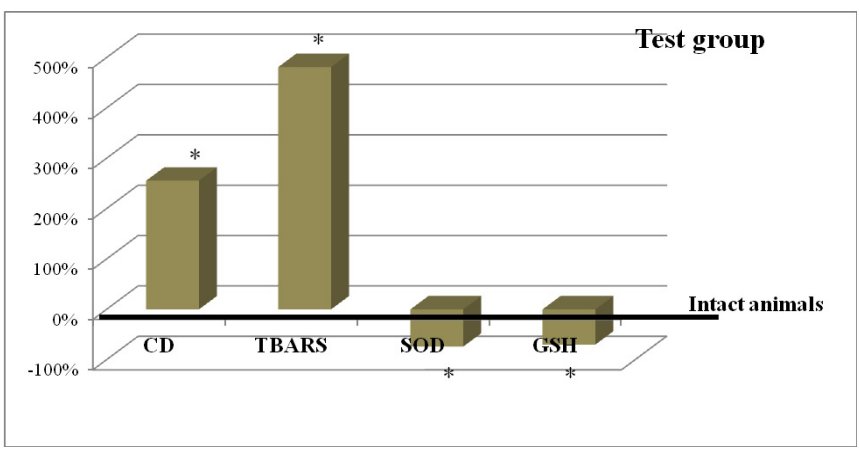

Fig. 1. The status of $L P O$ and $A O D$ in rats with inflammatory and degenerative damage of the soft tissues of the periodontium. $*_{-} P<0.05$ compared to intact animals.

In $\mathrm{TG}$, lymphokine production by $\mathrm{T}$ lymphocytes in LMIT with ConA and PHA was depressed by 1.64 and 1.58 times, respectively, compared to CG (Fig.2). In TG, PN and IPC it was increased a statistically significant amount (by 1.26 and 1.22 times), while PI decreased by 1.45 times compared to CG. The activity of oxygen-independent microbicidal systems of phagocytes estimated by LCT decreased by 1.16 times. In addition, indexes of the spontaneous NBT test and induced NBT test increased by 1.62 and 1.35 times, respectively, compared to CG (Fig.2).

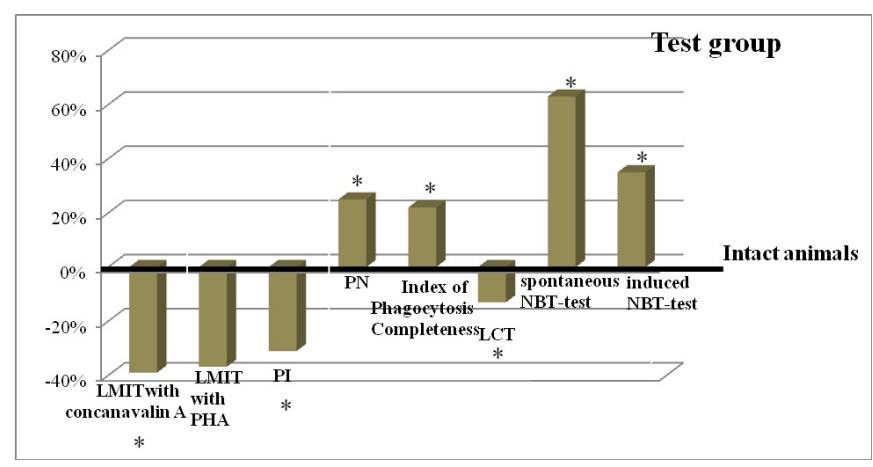

Fig. 2. Changes in immunological indicators in rats with inflammatory and degenerative damage of the soft tissues of the periodontium. ${ }^{*}-P<0.05$ compared to intact animals.

The results obtained showed that the modeling of inflammatory and degenerative damage to the periodontium in rats was characterized by significant changes, affecting both the LPO system and the immune response. Broken oxygen delivery under hypoxia in the tissues of the periodontium with the cascade of biochemical reactions, involving disturbances in the energy metabolism, formed the basis of this damage. ${ }^{(4)}$ Dramatic changes in cellular redox systems triggered the suppression of antioxidative defense in biological tissues and internal environments. ${ }^{(17)}$

The prooxidative/antioxidative cellular imbalance between the production of ROS and the ability of the biological systems' defense mechanisms to eliminate the cellular stress disturbances leads to a vicious circle, since OS reciprocally aggravates ROS production. OS acts as a leading pathogenetic factor of microcirculatory disorders. The disorganization of homeostatic mechanisms of the periodontal microcirculation has been identified as a cause of chronic tissue hypoxia of the periodontal complex, in which the processes of LPO of biomolecules are activated, leading to a violation of the structure and function of the periodontal biomembranes. ${ }^{(5,25)}$ Progression of periodontitis greatly depends on the state of the immune system, closely related to free radical oxidation, which determines the progression of inflammation as a typical pathological process. ${ }^{(4)}$ An LMIT conducted with various mitogens demonstrated the development of immunodeficiency in TG characterized by high activity of the inflammatory process.

Immune disorders in animals of TG were also supported by changes in the phagocytosis system reflected in decreased PI values. Low levels on the integral NBT test indicate disorders in the state of oxygen-dependent microbicidal systems of phagocytes. The assessment of the activity of oxygen-independent microbicidal systems of phagocytes in LCT also found disorders in the immune status of animals of TG. Thus, inflammatory and degenerative damage of the soft tissues of the periodontium in rats is accompanied by pronounced disorders in both the LPO system and the immune response.

\section{Competing Interests}

The authors declare that they have no competing interests.

\section{References}

1. Kassebaum NJ, Bernabé E, Dahiya M, Bhandari B, Murray CJ, Marcenes W. Global burden of severe periodontitis in 1990-2010: a systematic review and meta-regression.J Dent Res. 2014;93(11):1045-53. doi: 10.1177/0022034514552491. 2. GBD 2015 Disease and Injury Incidence and Prevalence Collaborators. Global, regional, and national incidence, prevalence, and years lived with disability for 310 diseases and injuries, 1990-2015: a systematic analysis for the Global Burden ofDisease Study 2015. Lancet. 2016;388(10053):15451602. doi: 10.1016/S0140-6736(16)31678-6.

3. Tonetti MS1,2, Jepsen S3, Jin L2, Otomo-Corgel J4. Impact of the global burden of periodontal diseases on health, nutrition and wellbeing of mankind: A call for global action. J Clin Periodontol. 2017;44(5):456-462. doi: 10.1111/ jcpe.12732. Epub 2017 May 8.

4. Bykova NI, Odol'skii AV, Grigoryan VA. [Indicators of cellular and humoral immunity in experimental inflammation of periodontal tissues]. Kuban Scientific Medical Bulletin. 2016;6:20-26. [Article in Russian].

5. Mokrenko YeV, Shabanov PD. [Modeling of inflammatory and degenerative damages of paradont tissue for assessment of drugs and food supplements effect]. Obzory po klinicheskoi farmakologii i lekarstvennoi terapii. 2013;11(2):57-59. [Article in Russian].

*Corresponding author: Marina A. Darenskaya, PhD, ScD. Scientific Centre for Family Health and Human Reproduction Problems, Irkutsk, Russia. E-mail: marina darenskaya@inbox.ru 
6. Sycheva YuA, Gorbacheva IA, Orekhova LYu, Grigor'eva LG, Chudinova TN. [The role of regional micro-circulation and metabolic disorders in pathogenesis of hypertensive disease and inflammantary diseases of the periodontium]. Periodontology. 2014;2(71):32-35. [Article in Russian].

7. Nath SG, Raveendran R. Microbial dysbiosis in periodontitis. J Indian Soc Periodontol. 2013;17(4):543-5. doi: 10.4103/0972-124X.118334.

8. Kolesnikova LI, Semyonova NV, Grebenkina LA, Darenskaya MA, Suturina LV, Gnusina SV. Integral indicator of oxidative stress in human blood. Bull Exp Biol Med. 2014;157(6):715-7. doi: 10.1007/s10517-014-2649-z.

9. Darenskaya MA, Grebenkina LA, Sholokhov LF, Rashidova MA, Semenova NV, Kolesnikov SI, Kolesnikova LI. Lipid peroxidation activity in women with chronic viral hepatitis. Free Radical Biology \& Medicine. 2016;100(S1):192. 10. Darenskaya MA, Rychkova LV, Kolesnikov SI, Gavrilova OA, Kravtsova OV, Grebenkina LA, Kolesnikova LI. Oxidative stress parameters in adolescent boys with exogenous-constitutional obesity. Free Radical Biology \& Medicine. 2017;112:129-130.

11. Kolesnikova LI, Kolesnikov SI, Darenskaya MA, Grebenkina LA, Nikitina OA, Lazareva LM, et al. Activity of LPO processes in women with polycystic ovarian syndrome and infertility. Bull Exp Biol Med. 2017;162(3):320-322. doi: 10.1007/s10517-017-3605-5.

12. Ershova OA, Bairova TA, Kolesnikov SI, Kalyuzhnaya OV, Darenskaya MA, Kolesnikova LI. Oxidative stress and catalase gene. Bull Exp Biol Med. 2016;161(3):400-3. doi: 10.1007/s10517-016-3424-0.

13. Bairova TA, Kolesnikov SI, Kolesnikova LI, Pervushina OA, Darenskaya MA, Grebenkina LA. Lipid peroxidation and mitochondrial superoxide dismutase- 2 gene in adolescents with essential hypertension. Bull Exp Biol Med. 2015;158(2):1814. doi: 10.1007/s10517-014-2717-4.

14. Kolesnikova LI, Darenskaya MA, Semenova NV, Grebenkina LA, Suturina LV, Dolgikh MI et al. Lipid peroxidation and antioxidant protection in girls with type 1 diabetes mellitus during reproductive system development. Medicina (Lithuania). 2015;51(2):107-111.
15. Darenskaya MA, Gavrilova OA, Rychkova LV, Kravtsova OV, Grebenkina LA, Osipova EV, et al. The assessment of oxidative stress intensity in adolescents with obesity by the integral index. International Journal of Biomedicine. 2018;8(1):37-41.

16. Kolesnikova LI, Kolesnikov SI, Korytov LI, Suslikova MI, Darenskaya MA, Grebenkina L.A, Kolesnikova L.R. Oxidative stress as a mechanism of reduced glucose absorption under conditions of immobilization stress. Bull Exp Biol Med. 2017;164(2):132-135. doi: 10.1007/s10517-017-3941-5.

17. Omarov IA, Bolevich SB, Savateeva-Lyubimova TN, Silina EV, Sivak KV. [Oxidative stress and comprehensive antioxidative energy correction in treatment of periodontitis]. Stomatology. 2011;1:10-17. [Article in Russian].

18. Kolesnikova LI, Kolesnikov SI, Romanova ED, Chkhenkeli VA, Darenskaya MA, Grebenkina LA, et al. Effect of Preparation Based on Trametes Pubescens Xylotroph Fungi on Lipid Peroxidation in the Blood of Experimental Animals under Conditions of Dark Stress. Bull Exp Biol Med. 2017;162(6):762-764. doi: 10.1007/s10517-017-3707-0.

19. Mokrenko EV, Shabanov PD. Therapy of inflammatory and degenerative damage of the smooth parodont tissue with tooth pastes in rats. Pediatr. 2015;6(2):81-86. [Article in Russian].

20. Kamyshnikov VS. Handbook on clinical and biochemical studies and laboratory diagnostics. Moscow; 2009. [in Russian]. 21. Potemkina EE, Pozdnyakova RZ, Manukyan LM. [Training Manuals for Laboratoty Clinical Immunology]. Moscow; 2003. [in Russian].

22. Nazarenko GI, Kishkun AA. [Clinical assessment of laboratory results]. Moscow; 2007. [Article in Russian].

23. Negative nitroblue tetrazolium reduction test. Available from: https://www.ncbi.nlm.nih.gov/medgen/336678

24. Khabriev RU. [Guidance on experimental (non-clinical) testing of new pharmaceutical substances]. Moscow; 2005. [Article in Russian].

25. Gumus P, Huseyinalemdaroglu B, Buduneli N. The role of oxidative stress in the interaction of periodontal disease with systemic diseases or conditions. Oxid Antioxid Med Sci. 2016;5(2):33-3817:18. 